\section{Capgras syndrome in substance-induced psychosis}

\author{
Bharat R. Sampathi, Anna Sofine, \\ John Alvarez, Robert Bota \\ University of California Irvine, Orange, \\ CA, USA
}

\begin{abstract}
Capgras Syndrome is a subcategory of delusional disorder. People affected by this syndrome believe that a close associate such as a friend or family member has been replaced by an identical imposter. This case report describes a 23-year-old woman with no prior psychiatric history, whom developed Capgras syndrome, via folie a deux, in the setting of poly-substance use. In this patient, a combination of Aripiprazole 10 $\mathrm{mg}$ daily and Escitalopram $10 \mathrm{mg}$ daily were effective in resolving symptoms. Clonazepam was utilized for anxiety and Omega-3 fatty acids $1 \mathrm{~g}$ for anti-oxidative effects. Further studies are needed to investigate the effects of a variety of causes and treatments for Capgras Syndrome.
\end{abstract}

\section{Introduction}

Delusional disorder refers to a condition in which an individual presents false beliefs, despite overwhelming counterevidence and improbability. Specifically, one must have one or more delusions for at least one month while failing to meet criteria for schizophrenia or any other psychotic disorder. Other than the direct impact of the delusion, behaviors are not bizarre or odd and functioning is usually uncompromised. A subcategory exists within delusional disorder known as a Capgras Syndrome. A person affected with this syndrome will believe that a close associate such as a friend or family member has been replaced by an identical imposter.

Multiple studies and reports have commented on Capgras Syndrome in the setting of various neurological and neurodegenerative diseases. A 2014 study found it to be prevalent in first-episode psychotic disorders (schizophreniform psychosis 50\%, brief psychosis $34.8 \%$, or unspecified psychosis $23.9 \%$ ). ${ }^{1}$ Another study determined that Electroconvulsive therapy followed by mood-stabilizer and antipsychotic drug combination led to the permanent, effective control of long-standing Capgras in young women. ${ }^{2}$ For the overarching delusion disorder, the most studied first-line treatment regimen involves a combination of second generation antipsychotics and psychotherapy. It has also been emphasized that comorbid conditions such as anxiety, depression, and substance abuse must be simultaneously treated.

Induced delusional disorder, as named in ICD-10, or Folie a deux, also known as shared psychotic disorder in DSM-IV, which has been removed from DSM-V, is described as a phenomenon seen when a delusional belief is transmitted from one individual to another. ${ }^{3}$ This type of delusion is rare and is mostly described via case reports. Some reported risk factors developing a delusion are female gender, suggestibility, suspiciousness, and passivity. ${ }^{4}$

This case report describes a patient with no prior psychiatric history whom developed Capgras Syndrome, via folie a deux, in the setting of poly-substance use.

\section{Case Report}

A 23-year-old female with no previous psychiatric diagnosis presented to the emergency department (ED) brought in by mother for extremely agitated behavior. Upon interview, patient endorsed methamphetamine and marijuana use the prior night as well as attending a music festival a week prior where she used cocaine and multiple forms of 3,4-Methylenedioxyme-thamphetamine (MDMA). She mentioned that she had not gotten any sleep the previous night and usually sleeps 8 hours per night. She was dysphoric, paranoid, endorsed auditory hallucinations, and demonstrated extreme suspicion of the family pictures on the walls of her parent's home. She repeatedly told her mother that "the pictures are not real", and directly referred to her family as imposters. Patient endorsed symptoms of depression over the past six months, but denied low energy, lack of sleep, poor concentration, guilt, or change in appetite. After stating that she would shoot herself if she had a gun due to paranoia about her family being imposters she was ultimately admitted voluntarily due to high risk for imminent suicide with significant risk factors including recent drug use, impulsivity, disconnect from reality due to psychosis, and current suicidal ideation.

She was started on Aripiprazole $10 \mathrm{mg}$ daily for psychosis and Clonazepam $0.5 \mathrm{mg}$ twice a day as needed for anxiety. Over the course of several days insight and judgement improved and she appeared more linear and logical, able to attribute her polysubstance abuse as a probable instigator to her psychosis. As she continued to endorse intermittent suicidal ideations,
Correspondence: Robert G Bota, Department of Psychiatry, University of California Irvine, 101 City Drive

Orange, CA 92617, USA.

Tel.: +1.229.815.0219 - Fax: +1.714.456.2056.

E-mail: rgbota@yahoo.com

Key words: Capgras Syndrome, neurological and neurodegenerative diseases, substanceinduced psychosis.

Contributions: the authors contributed equally.

Conflict of interest: the authors declare no potential conflict of interest.

Funding: none.

Received for publication: 2 August 2018. Accepted for publication: 2 August 2018.

This work is licensed under a Creative Commons Attribution-NonCommercial 4.0 International License (CC BY-NC 4.0).

(C) Copyright B.R. Sampathi et al., 2018

Licensee PAGEPress, Italy

Mental Illness 2018; 10:7807

doi:10.4081/mi.2018.7807

Escitalopram $10 \mathrm{mg}$ daily for depression and Omega-3 fatty acids $1 \mathrm{~g}$ daily for mood were added. Clonazepam was discontinued over the next few days.

She then endorsed that the night prior to admission; she had visited a bar and met a man whom she later accompanied home. At his home, the patient used marijuana and methamphetamine after which the man became paranoid and showed the patient a picture of him on his phone and stating that the picture was not him. Rather, it was an imposter who had been following him. She then became paranoid regarding imposters of both herself and her family. When she returned home, she was immediately suspicious of her mom and believed the pictures on the wall were all "fake." It took several days of Aripiprazole and daily visits from close family members to assuaged her concerns that her family members were imposters and that the home pictures were "fake". As her paranoia resolved she became pleasant and appropriately engaging. Her suicidal ideation resolved over several days, prior to the timeline which Escitalopram would have played much of a therapeutic role. Therefore, it is probable that suicidal ideation was an indirect result of acute psychosis as well. Within a week, she was found to no longer be a danger to self, danger to others, or gravely disabled, and was discharged home. 


\section{Discussion and Conclusions}

There are limited studies on the efficacy of treatments for Capgras syndrome. In this patient, a combination of Aripiprazole 10 $\mathrm{mg}$ daily and Escitalopram $10 \mathrm{mg}$ daily were effective in resolving symptoms. Clonazepam was utilized for anxiety and Omega-3 fatty acids $1 \mathrm{~g}$ for anti-oxidative effects.

Our team determined the most likely provocation of her Capgras syndrome was polysubstance use with resulting paranoia. She mentioned her paranoia was acutely exacerbated when the stranger she was with questioned the validity of his own picture of himself. She then became highly suspicious of her mom and the family pictures at her home, via what appears to have been a folie a deux response. While it has been documented that second-generation antipsychotics have been effective in treating delusional disorder, it is unclear whether this patient's resolution of Capgras Syndrome was a result of the elimination of THC and methamphetamine from her body or the effect of Aripiprazole. It is postulated that a combination was required to elicit such profound dislodging of the fixed Capgras delusion. It is likely that recidivism of substance would result in relapse of symptoms. Further studies may investigate the effects of a variety of causes and treatments for Capgras Syndrome.

\section{References}

1. Salvatore $\mathrm{P}$, Bhuvaneswar C, Tohen M, et al. Capgras' syndrome in first-episode psychotic disorders. Psychopathology 2014;47:261-9.

2. Rapinesi C, Kotzalidis GD, Del Casale A, et al. Treatment-resistant, five-year long, postpartum-onset Capgras episode resolving after electroconvulsive therapy. Int $\mathbf{J}$ Psychiatry Med 2015;49:227-34.

3. Dewhurst K, Todd J. The psychosis of association--folie a deux. J Nerv Ment Dis 1956;124:451-9.

4. Jolfaei AG, Isfahani MN, Bidaki R. Folie à deux and delusional disorder by proxy in a family. J Res Med Sci 2011;16: S453-5. 